\title{
Nature des dommages causés par les piqûres alimentaires des punaises (Heteroptera) sur les boutons floraux et les capsules du cotonnier au Togo
}

\author{
Panawé TOZOOU ${ }^{1 *}$, Wiyao POUTOULI ${ }^{1}$, Koffi KOBA ${ }^{2}$, Nafadjara A NADIO ${ }^{2}$, \\ Magnim E. BOKOBANA ${ }^{2}$, Mamatchi MELILA ${ }^{4}$, Pikassalé AKANTETOU ${ }^{3}$, \\ Bassarou AYEVA ${ }^{3}$, Bètibètè BONFOH ${ }^{3}$ et Amen Y. NENONENE ${ }^{2}$ \\ ${ }^{1}$ Laboratoire de Biologie Animale et de Zoologie, Faculté des Sciences, Université de Lomé, \\ B.P. 1515 Lomé, Togo. \\ ${ }^{2}$ Unité de Recherche sur les Agro-Ressources et la Santé Environnementale, Ecole Supérieure d'Agronomie, \\ Université de Lomé, BP. 20131, Lomé, Togo. \\ ${ }^{3}$ Institut Togolais de Recherche Agronomique (ITRA), BP 1163, Lomé, Togo. \\ ${ }^{4}$ Laboratoire de Biochimie Appliquée à la Nutrition et à l'Alimentation, Département de Biochimie/Nutrition, \\ Faculté des Sciences, Université de Lomé, Togo. \\ *Auteur correspondant, E-mail: panawetozoou@yahoo.fr; Tel: (00228)91668894
}

\section{RESUME}

En culture cotonnière au Togo, Les Hétéroptères constituent un facteur entomologique important par rapport à leur densité et les dommages qu'ils causent. Si leur rôle parasitaire peut sembler secondaire par rapport celui des Lépidoptères, il est cependant loin d'être négligeable. La protection contre les Lépidoptères ayant atteint une efficacité valable, il apparait désormais que l'on doive compter avec les Hétéroptères pour l'amélioration de la lutte insecticide. Ainsi, dans le but d'améliorer les rendements, il est nécessaire de connaître la nature des dommages causés par les Punaises. Pour y arriver, des boutons floraux et des capsules du cotonnier ont été infestés par 4 espèces de Mirides (Creontiades pallidus, Megacoelum apicale, Taylorilygus arboreus et Helopeltis schoutedeni). Toutes les espèces de Mirides, à l'exception de $H$. schoutedeni ont piqué les boutons floraux pour se nourrir du contenu des étamines. Ces piqûres ont provoqué des nécroses sur les pétales et les étamines qui ont été totalement ou partiellement détruites. Ces dommages ont développé sur les boutons floraux le phénomène d'abscission. Par contre, sur les jeunes capsules infestées, les résultats ont montré que les dégâts causés par $H$. schoutedeni ont été externes et se sont présentés sous forme de nécroses en cuvettes ou chancres. Les piqûres des autres espèces de Mirides sur les jeunes capsules, se sont traduites par un écoulement résineux extérieur et une formation d'excroissances sur la face interne des carpelles.

(C) 2015 International Formulae Group. All rights reserved.

Mots clés : Cotonnier, boutons floraux et capsules, Mirides, piqûres, dommages.

\section{INTRODUCTION}

Au Togo, la culture cotonnière connaît une extension remarquable depuis que la variété «Allen»(Gossypium hirsutum L.) a été introduite en 1964. Mais, de nombreux travaux génétiques ont permis la mise en place 
en 1985 de la variété STAM (Station Anié Mono) actuellement (STAM 129). Présentement, l'aire d'extension géographique de cette culture recouvre plus de $80 \%$ du territoire national et est conséquente de la diversité des groupes de déprédateurs. Cette culture est de par le monde celle qui héberge un nombre important de ces déprédateurs qui occasionnent des dégâts multiples aux divers organes des plants tout au long de leur cycle de développement (Hargreaves, 1948 ; Dième, 1980).

De tous les organismes nuisibles à la culture cotonnière, la faune entomologique est la plus importante. En effet, Hargreaves (1948) a inventorié sur cette culture 1326 espèces d'insectes dont 482 en Afrique au Sud du Sahara. Au Togo, Poutouli (1994) a inventorié sur cette culture 56 espèces d'Hétéroptères phytophages. Actuellement, plus de 7000 espèces de Mirides sont décrites et cette famille constitue la plus importante des Hétéroptères (Delvare et Aberlenc, 1989). Si la plupart des Mirides sont phytophages, nombreux d'entre eux sont prédateurs d'autres insectes (Cadou, 1994). L'abscission des organes floraux et capsulaires, est un phénomène longtemps dévolué aux seuls facteurs physiologiques. Mais, les travaux d'Ewing (1929) ont montré le rôle important de l'entomofaune dans l'abscission de ces organes. Le rôle réel des Mirides dans le phénomène d'abscission des boutons floraux du cotonnier a été élucidé par de nombreux travaux (Tugwell et al., 1976 ; Mauney et Henneberry, 1979 ; Mauney, 1984 ; Leigh et al., 1988). Au Togo, Les Hétéroptères constituent de plus en plus un facteur entomologique important par rapport à leur densité et les dommages qu'ils causent au cotonnier et aux autres plantes cultivées. Chaque année, des dégâts importants sont enregistrés: chute des organes florifères, piqûres des organes végétatifs et des capsules vertes, pourritures internes. Cependant, l'impact de ces derniers est mal connu. Aussi, plusieurs espèces d'Hétéroptères phytophages ont été signalées comme étant nuisibles à la culture cotonnière suite à leurs piqûres alimentaires sur les différents organes et principalement sur les organes fructifères (Poutouli, 1994). Pour connaître les niveaux d'infestation et prendre en considération ces ravageurs, des études particulières devraient être entreprises en vue de décrire les dégâts de chaque espèce déprédatrice. Le présent travail a été réalisé, dans ce cadre, afin de contribuer à l'amélioration des rendements de la culture cotonnière et a pour but d'identifier les dommages en conditions d'infestation artificielle.

\section{MATERIEL ET METHODES Zone d'étude}

Les travaux ont été réalisés à la station du Centre de Recherche Agronomique Savanes Humides (CRA-SH) d'Anié à Kolocopé à environ $250 \mathrm{~km}$ au Nord de Lomé (Figure 1). Le CRA-SH est situé entre $7^{\circ} 49^{\prime} \mathrm{N}$ et $1^{\circ} 20^{\prime} \mathrm{E}$ dans la région des Plateaux. C'est une zone de plaines au centre du Togo parcourue par des forêts sèches, une savane et des galeries forestières avec présence chaque année de feux de brousse. Elle jouit d'un climat subtropical du type Guinéen à quatre saisons (2 saisons de pluie et 2 saisons sèches).

\section{Matériel végétal}

Le matériel végétal utilisé est le cotonnier Gossypium hirsutum L., variétés STAM 129, du nom de la station (Station Anié Mono) à Kolokopé où elles sont créées et vulgarisées au Togo et ailleurs dans la sousrégion ouest africaine.

\section{Infestation artificielle des boutons floraux}

Cette opération a été réalisée dans les parcelles ne recevant aucun traitement insecticide pendant toute la saison. 
Des manchons cylindriques en nylon, de maille $1 \mathrm{~mm}$, de dimensions variables ont été confectionnés à cet effet. Des plants de cotonnier sont choisis au hasard pour isoler les organes apparemment sains. Les branches qui portent des boutons floraux, sont introduites dans les manchons (Photo 1) dont l'unique ouverture est maintenue fermée à l'aide d'attache-lettres pendant cinq jours. $\mathrm{Au}$ sixième jour, un examen visuel des organes a permis d'éliminer les fleurs et les organes qui étaient tombés ou qui présentaient des dégâts extérieurs visibles. Les organes sains, toujours maintenus dans les manchons, ont été répartis en 2 lots : un lot témoin constitué de 40 boutons floraux, n'a reçu aucun Miride, un lot de 160 organes foraux, infestés, a été réparti en quatre groupes de 40 boutons floraux chacun. Les organes de chaque groupe ont été infestés par un adulte (mâle ou femelle) des Mirides sélectionnés.

\section{Infestation artificielle des capsules}

L'isolement des capsules, apparemment saines, s'est fait dès la chute de la corolle et pendant trois semaines. Un examen visuel a permis de voir si une capsule porte, ou non, des dégâts extérieurs visibles. Les 40 capsules saines, toujours maintenus dans les manchons (Photo 1), ont été réparties en 8 groupes de 5 capsules chacun. Les capsules de chaque groupe sont infestées par un adulte (mâle ou femelle) des espèces de punaises sélectionnées.

Il faut noter que, dans le cas de Helopeltis schoutedeni, ce sont les larves et les adultes qui ont été utilisés.

Pour l'infestation, les punaises (Tableau 1) ont été capturées sur des plantes hôtes (maïs, niébé, pois d'angole, soja et sorgho) autres que le cotonnier. Les différentes espèces de punaises captées ont fait l'objet d'un suivi au champ sur les cotonniers par observations visuelles et leurs comportements ont été décrits. Une fois capturées, les punaises ont été conservées à jeun au laboratoire pendant 24 heures avant leur introduction dans les manchons au champ. Le séjour des punaises dans les manchons a été de 5 jours. Après ce délai, les punaises ont été retirées, les organes étant toujours maintenus isolés pendant 5 jours encore avant d'être récoltés pour la dissection et l'observation au laboratoire sous une loupe binoculaire. Au moment de la récolte, tous les organes déjà tombés dans les manchons ont été comptés pour chaque espèce de punaises.

Tableau 1 : Punaises utilisées pour les infestations artificielles.

\begin{tabular}{lc}
\hline Familles & Adultes de Punaises \\
\hline Creontiades pallidus (Rambur, 1840) (Photo 2) \\
Megacoelum apicale (Reuter, 1882) (Photo 3) \\
Taylorilygus arboreus (Taylor, 1947) (Photo 4) \\
Helopeltis schoutedeni (Reuter, 1906) (Photos 5 et 6) \\
\hline
\end{tabular}




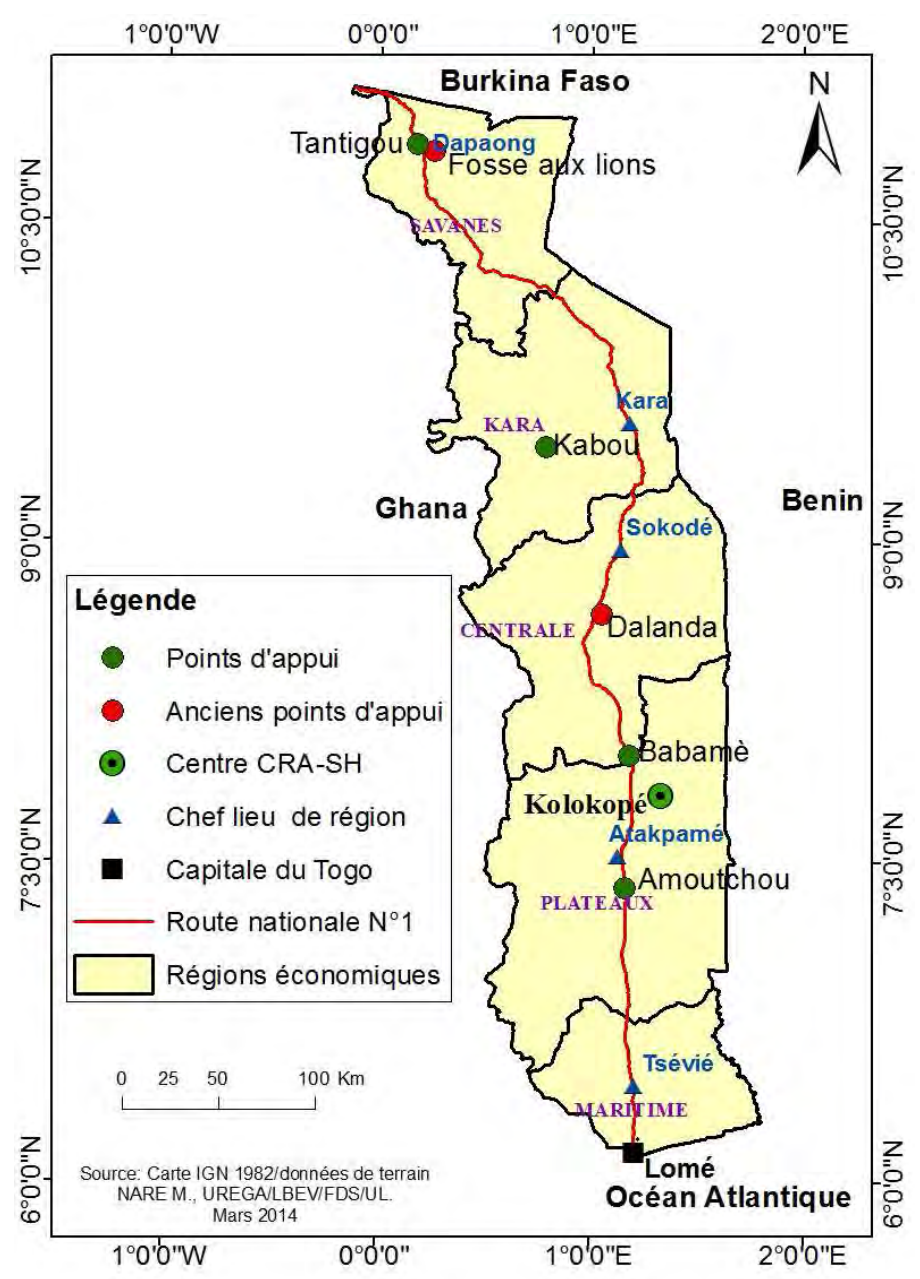

Figure 1 : Indication du Centre de Recherche Agronomique Savanes Humides (CRA-SH) d'Anié à Kolocopé (Togo) et de ses différents points d'appui.



Photo 1 : Branche fructifère portant les boutons floraux dans le manchon. 


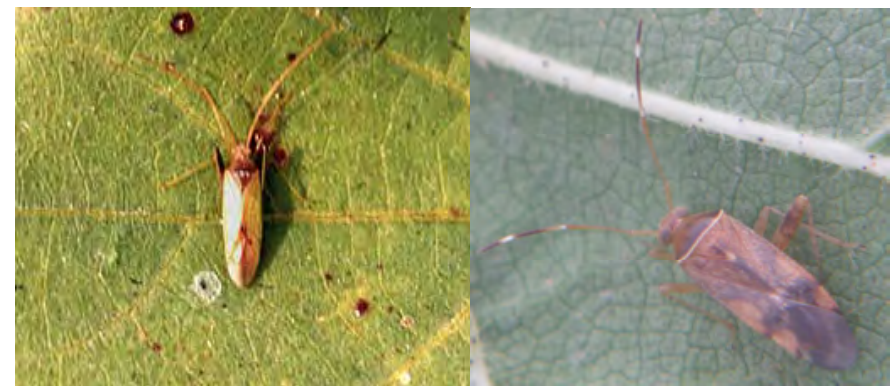

Photo 2 : Creontiades pallidus (Rambur, 1840).

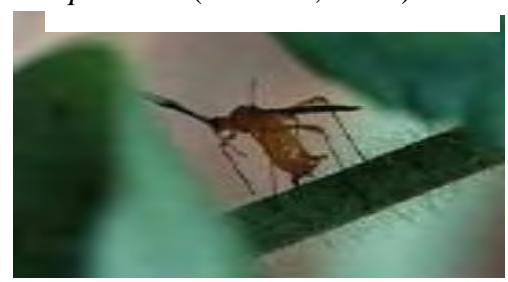

Photo 5 : Helopeltis schoutedeni (Reuter, 1906) (adulte) (Poutouli et al., 2012). apicale (Reuter, 1882).

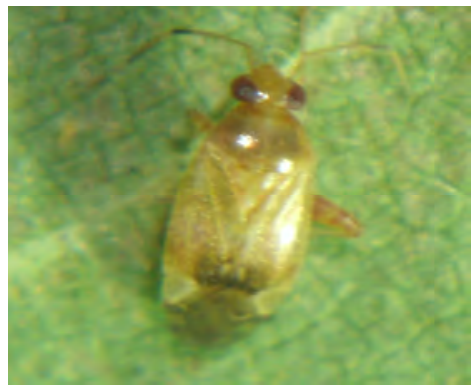

Photo 4 : Taylorilygus arboreus (Taylor, 1947).



Photo 6 : Helopeltis schoutedeni (Reuter, 1906) (larve) (Poutouli et al., 2012).

\section{RESULTATS}

Il n'y a pas eu de mortalité des insectes introduits dans les manchons et des femelles ont pondu. Le microclimat créé dans ces enceintes leur a été donc favorable.

\section{Dégâts observés sur les boutons floraux infestés artificiellement}

Les piqûres alimentaires des punaises sur les boutons floraux infestés sont observables par des nécroses brunes sur les pièces protectrices (Photo 7) et sur les étamines (Photo 8).

\section{Boutons floraux dans le témoin}

$\mathrm{Au}$ moment de leur récolte pour la dissection, 8 boutons floraux étaient déjà tombés dans les manchons (Tableau 2). Après dissection des 40 boutons floraux témoins, aucun d'entre eux n'a présenté ni piqûres ni nécroses sur les pièces protectrices et sur les étamines. Ils ont été qualifiés de boutons floraux sains (Photo 9) et ont présenté des étamines normales (Photo 10). L'abscission des 8 boutons floraux au moment de la récolte peut être d'origine physiologique donc non liée à une cause entomologique.

\section{Boutons floraux infestés par $C$. pallidus}

$\mathrm{Au}$ moment de leur récolte pour dissection, 29 boutons floraux étaient déjà tombés dans les manchons (Tableau 2). Après dissection, 39 boutons floraux au total ont présenté des piqûres et des nécroses brunes sur les parties suivantes du bouton floral:

- les pétales : le nombre de ces points de nécroses a été variable d'où la preuve que ces boutons floraux ont été piqués plusieurs fois. Ces points de nécroses sur les pétales débouchent directement sur des zones précises de la masse staminale.

- les étamines : on a pu observer un nombre variable de zones nécrosées. Dans ces zones, les anthères ont été rétrécies donc vidées de leur contenu et virent au brun marron; on ne pouvait plus y reconnaître anthères et filets. Les étamines ont été donc détruites. Sur les jeunes boutons floraux, on a constaté, souvent, la destruction totale de la masse staminale et il ne restait qu'une masse 
gélatineuse. Mais, sur les boutons floraux plus âgés, c'est plusieurs de ces zones de destruction qui ont été observées et réparties sur l'ensemble de la masse staminale. Ces dégâts sont plus concentrés dans les $2 / 3$ supérieurs de la masse staminale et il a été difficile de faire une distinction entre les piqûres des mâles et celles des femelles de ce Miride.

\section{Boutons floraux infestés par $M$. apicale}

$\mathrm{Au}$ moment de leur récolte pour la dissection, 32 boutons floraux étaient déjà tombés dans les manchons (Tableau 2). Après dissection, 37 boutons floraux ont présenté des piqûres et des nécroses sur les pétales et sur les étamines et étaient identiques à celles décrites plus haut. Nous n'avons remarqué aucune différence entre les piqûres des mâles et celles des femelles ni entre les deux espèces.

\section{Boutons floraux infestés par $T$. arboreus}

$\mathrm{Au}$ moment de leur récolte pour la dissection, 15 boutons floraux étaient déjà tombés dans les manchons (Tableau 2). Après dissection, 28 boutons floraux ont présenté des piqûres et des nécroses sur les pétales et sur les étamines. Dans le cas de cette espèce, les piqûres ont été plutôt localisées à la base des boutons floraux, dans leur tiers inférieur. Ainsi donc, les piqûres ont été faites sur le calice sans aucune trace visible. Les zones de destructions des étamines ont été observées à la base de la masse staminale.

\section{Boutons floraux infestés par $\boldsymbol{H}$. schoutedeni}

$\mathrm{Au}$ moment de leur récolte pour la dissection, 5 boutons floraux étaient déjà tombés dans les manchons (Tableau 2). Après dissection des 40 boutons floraux infestés par ce Miride, aucun organe n'a présenté des piqûres ni nécroses sur les pétales et sur les étamines. Les étamines observées sont entières avec des filets et des anthères normaux c'est-à-dire turgescents et de couleur jaune. La chute des 5 boutons floraux dans les manchons aurait une origine différente des facteurs entomologiques.
Aucune différence n'a été observée entre les symptômes causés par les piqûres de chacune des trois espèces de Mirides sur les boutons floraux. Les nécroses observées sont identiques à celles qui sont observées lors de l'analyse des organes tombés aux champs.

\section{Infestation artificielle des capsules}

Les dégâts observés sur les capsules infestées ont été externes ou internes selon l'espèce déprédatrice.

\section{Les capsules infestées par $\boldsymbol{H}$. schoutedeni.}

Les piqûres occasionnées par ce ravageur ont causé des dégâts externes. Les symptômes se sont présentés sous forme de nécroses en cuvettes appelées chancres. Ces nécroses ont été plus concentrées au sommet des capsules et surtout au niveau des lignes de sutures intercarpellaires (Photo 11); ce qui a provoqué une ouverture prématurée de l'organe piqué (Photo 12)

Les capsules infestées par M. apicale, $T$. arboreus et $C$. pallidus

Les dégâts causés par ces trois espèces ont été différents de ceux de $H$. schoutedeni. En effet, leurs piqûres ont laissé la capsule apparemment saine. Mais l'observation à la loupe binoculaire a montré de petites cuvettes dont le centre était occupé par un exsudat noir métallique dû probablement à l'écoulement de résine. Ces cuvettes sont plus étendues chez $T$. arboreus et M. apicale que chez C. pallidus.

Ces cuvettes correspondent, sur la face interne des carpelles, à des formations néoplasmiques ou à des zones colorées (Photo 13). La formation de ces néoplasmes serait fonction de la profondeur de la piqûre car une piqûre superficielle n'entraînerait qu'un simple écoulement de résine. Dans le cas de $T$. arboeus et de $C$. pallidus, lorsque les piqûres ont été nombreuses, des cas de croissance inégale entre les différentes loges ont été constatés. Des cas de coloration des fibres ont été observés pour les trois espèces de Mirides (Photo 13). 
Tableau 2 : Effets de l'infestation de 40 boutons floraux selon l'espèce de Miride utilisée.

\begin{tabular}{lccccc}
\hline & \multicolumn{5}{c}{ Les espèces de Mirides utilisées } \\
\cline { 2 - 6 } Boutons floraux & C. pallidus & M. apicale & T. arboreus & H. schoutedeni & Témoin \\
\hline Abscission à la récolte & $29\left(0^{*}\right)$ & $32\left(3^{*}\right)$ & $15\left(2^{*}\right)$ & $\left(5^{*}\right)$ & $\left(8^{*}\right)$ \\
Piqûres + nécroses & 39 & 37 & 28 & 0 & 0 \\
(\%) de piqûres & 97 & 92 & 70 & 0 & 0 \\
\hline
\end{tabular}

*Boutons floraux tombés sans symptômes de piqûres.



Photo 7 : Nécroses brunes des piqûres nutritionnelles des mirides sur les pétales du bouton florale.

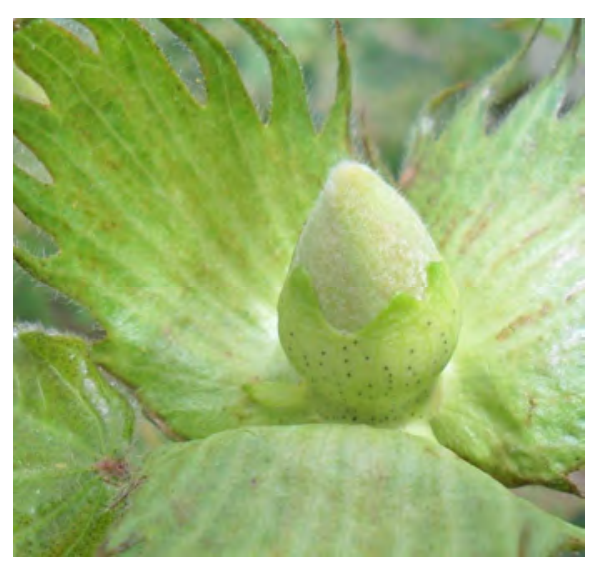

Photo 9 : Bouton floral sain du cotonnier.

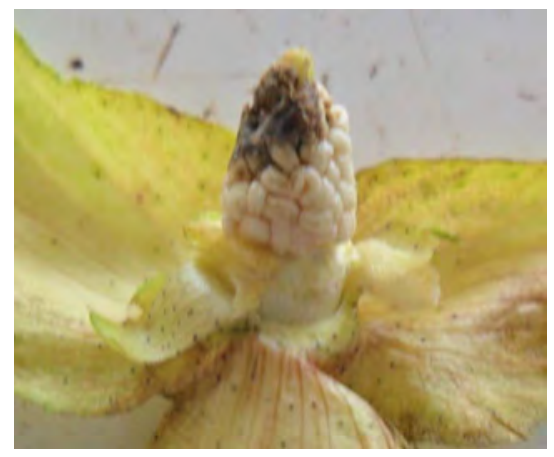

Photo 8 : Nécroses brunes des piqûres nutritionnelles des mirides sur les étamines.

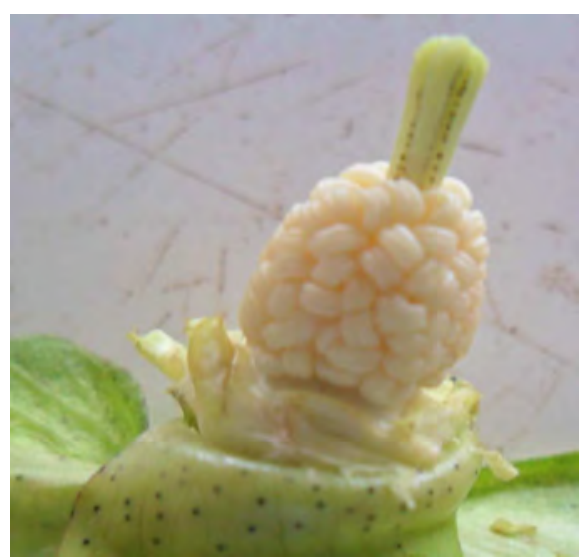

Photo 10 : Bouton floral sain disséqué montrant les étamines normales. 


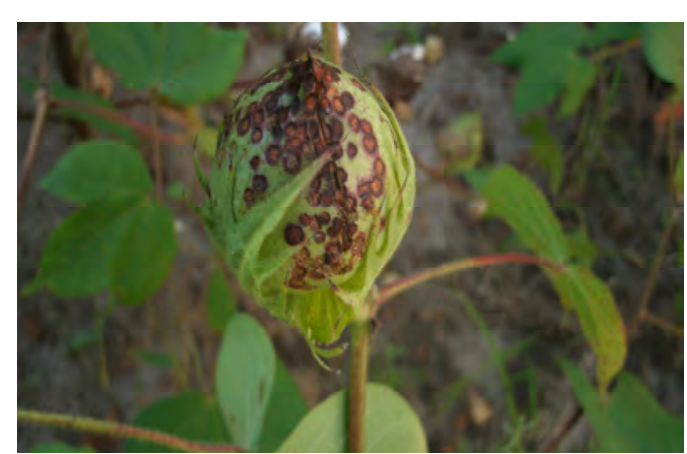

Photo 11 : Chancre sur capsule causé par $H$. schoutedeni.

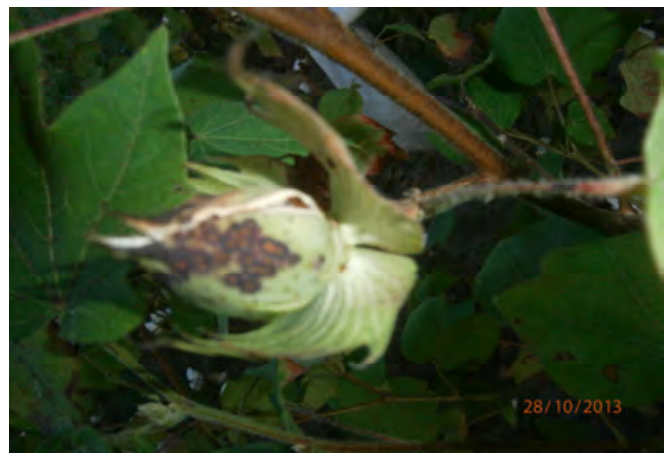

Photo 12 : Chancre causés par $H$. schoutedeni sur capsule et ouverture prématurée de celle-ci.

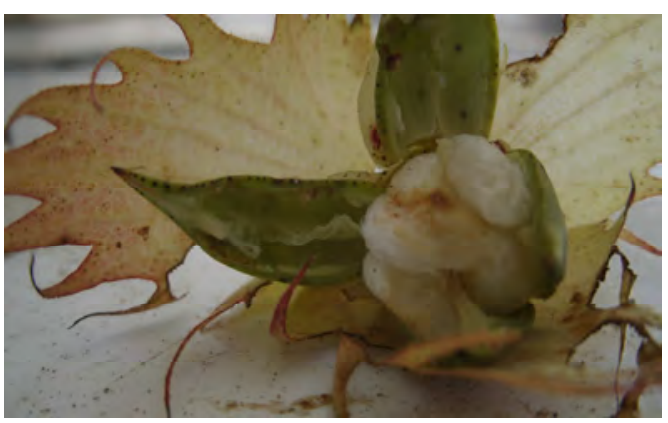

Photo 13 : Dommages internes des piqûres des mirides sur capsule.

\section{DISCUSSION}

Les punaises sont capables de causer des dommages aux divers organes des plants de cotonniers (Cadou, 1994). La technique d'infestation artificielle des organes floraux utilisée dans cette étude a permis d'avoir une idée sur les symptômes causés par certains ravageurs des cultures. Les symptômes observés et décrits sur les boutons floraux sont identiques à ceux observés par Pack et Tugwell (1976). Cependant, les espèces de Mirides les plus citées par des auteurs sont du genre Lygus, Pseudatomoscelis non identifiés au Togo. L'espèce bien connue au Togo est $C$. pallidus (Poutouli, 1994). Aussi, en Côte d'Ivoire, au Congo, au Soudan ou en Syrie, $C$. pallidus est bien connue pour son rôle dans l'abscission des boutons floraux et jeunes capsules ; c'est pour cette raison que l'espèce est dénommée en Anglais «the shedder bug »
(Paerson et al., 1958 ; Stam, 1987). En Egypte, le terme de «Cotton square shedder » est attribué à cette espèce pour bien caractériser son effet (Soyer, 1942). Pareils symptômes observés sur les boutons floraux, permettent de dire que les Mirides étudiés ont une part de responsabilité dans l'abscission de plusieurs organes enregistrés chaque année. Ceci, parce que, ces symptômes sont identiques à ceux observés lors de l'analyse sanitaire des organes tombés. Au Togo, $C$. pallidus, $T$. arboreus et $M$. apicale attaquent les boutons floraux en se nourrissant du contenu des anthères, en développement, qui se rétrécissent et virent au jaune brun noir au lieu d'être turgescentes et crèmes transparentes. Ces piqûres alimentaires ont pour conséquence la chute des boutons floraux piqués. Mais il est apparu impossible de distinguer, sur la base des dommages, les 
différentes espèces de Mirides responsables de l'abscission des boutons floraux. Les piqûres alimentaires des Mirides causent des dommages mécaniques aux boutons floraux; ce qui crée un stress physiologique et provoquerait leur abscission. De plus, les piqûres des Mirides ont aussi une action dans la balance hormonale (Auxine, Ethylène) (Tingey et al., 1977 ; Burden et al., 1989). En effet, l'aptitude de l'éthylène à inhiber le transport de l'auxine est bien connue, de même que celle de l'auxine à retarder ou à empêcher l'abscission (Beyer et Morgan, 1971). Aussi, la présence dans la salive des Mirides, de l'Acide 1-carboxylique 1-aminocyclopropane (ACC), précurseur de l'éthylène est évoquée par Burden et al. (1989). Cette hormone végétale dans la salive des Mirides est d'origine alimentaire et non héréditaire car de nombreux travaux réalisés sur les œufs des Mirides n'ont pas permis de déceler sa moindre trace (Burden et al., 1989).

Selon Taylor (1945), la salive des Mirides (Lygus) est acide et la décoloration des tissus peut être due à une destruction de la cloison médiane des parois cellulaires. Les dégâts sont donc vraisemblablement d'abord mécaniques, la décoloration des tissus est due secondairement à une action toxique.

Si les résultats de ces auteurs suggèrent que le Miride peut être capable de changer la balance des phytohormones pendant l'alimentation, une utilisation éventuelle des phytohormones marquées serait souhaitable pour confirmer cette hypothèse.

Sur les capsules, les dégâts engendrés par H. schoutedeni appelés chancres, ont été décrits par Leroy (1936). Les piqûres des autres Mirides sont responsables de l'écoulement de la résine sur les capsules caractérisé par un exsudat noir métallique. Ces symptômes ont été déjà décrits par Soyer (1942) Selon ces auteurs, Creontiades pallidus paraît rechercher les cellules glandulaires ; ce qui entraîne, après la piqûre, l'exsudation d'une fine gouttelette brune résineuse des cellules abîmées marquant ainsi, une auréole sombre sur 2 à $3 \mathrm{~mm}$ de diamètre.
Ces taches sont disséminées sur toute la capsule mais quelquefois un ou deux carpelles seulement sont concernés.

\section{Conclusion}

Les dommages des piqûres alimentaires des Mirides (Hétéroptères) ont été identifiés sur les boutons floraux et les capsules des cotonniers. Il est donc facile de faire une distinction entre les boutons floraux dont l'abscission est d'origine entomologique ou non. Pour cela, une dissection suivie d'une observation des pétales et des étamines a été indispensable. Les ponctions alimentaires des Mirides sur les boutons floraux ont laissé observer des nécroses sur les pétales (probablement dues au passage des stylets), des anthères totalement nécrosées ou partiellement détruites. Au Togo, les Mirides C. pallidus, $M$. apicale, T. arboreus sont les principaux insectes responsables de ces symptômes et donc de l'abscission des boutons floraux. Cependant, aucune différence n'a été faite entre les symptômes causés par chaque espèce de Mirides étudiée. Sur les capsules infestées artificiellement, l'essentiel des dégâts causés par $H$. schoutedeni ont été externes et se sont présentés sous formes de nécroses en cuvettes ou chancres. Les piqûres de $C$. pallidus, $M$. apicale, T. arboreus, sur les capsules, se sont traduites par un écoulement résineux extérieur et une formation d'excroissances sur la face interne des carpelles. Les niveaux des dégâts devraient être évalués et une densité seuil devrait être fixée afin d'envisager un traitement approprié contre ces Mirides pendant la période de la formation des organes floraux.

\section{REFERENCES}

Beyer EMJ-R, Morgan PW. 1971. Abscission: The role of ethylene modification of auxin transport. Plant physiology, 48: 208-212.

Burden BJ, Morgan PI, Sterling WL. 1989. Indoleacetic acid the ethylene precursor, Acc, in the cotton fleahopper (Hemiptera: 
Miridae) and their role in cotton square abscission”. Ann. Entomol. Soc. Am., 82: 476-480.

Cadou J. 1994. Les Miridae du cotonnier en Afrique et Madagascar. Série les déprédateurs $\mathrm{du}$ cotonnier en Afrique tropicale et dans le reste monde $n^{\circ} 8,74 p$.

Delvare G. Eberlenc HP. 1989. Les insectes d'Afrique et d'Amérique Tropicale. Clés pour la Reconnaissance des Familles. CIRAD, Montpellier, 298p.

Dième E. 1980. Effets des traitements insecticides sur les fluctuations du peuplement entomologique des cotonniers en Côte d'Ivoire centrale. Thèse de $3^{\text {ème }}$ cycle, Université de ParisSud, 171p.

Ewing KP. 1929. Effects on the cotton plant of the feeding of certain Hemiptera of the family Miridae. J. Econ. Entomol., 22: 761-765.

Hargreaves H. 1948. List of recorded cotton insect in the world. Commonw. Inst. Entomol., 50p.

Leigh TF, Kerby TA, Wynholds PF. 1988. Cotton square damage by the plant bug, Lygus hersperus (Hemiptera: Heteroptera: Miridae), and abscission rates. J. Econ. Entomol., 88: 1328-1337.

Leroy JV. 1936. Observations Relatives à Quelques Hémiptères du Cotonnier (Série 10). Institut National pour l'Etude Agronomique du Congo Belge (INEAC): Congo ; 3-20.

Mauney JR. 1984. Cotton square shedding: why they fall; what it means to yields. Crops and Soils Magazine, 1984: 20-22.

Mauney JR, Henneberry TJ. 1979. Identification of damage symptoms and patterns of feeding of plant bugs in cotton. J. Econ. Entomol., 72: 496-501.
Pack TM, Tugwell P. 1976. Clouded and tarnished plant bugs on cotton: a comparison of injury symptoms and damage on fruit parts. Ark. Agr. Exp. Stat. Report, 226: $17 \mathrm{p}$.

Poutouli W. 1994. Contribution à l'étude des Hétéroptères associés à la rotation culturale maïs- cotonnier- niébé au Togo. Thèse de docteur d'Université, Université Paris VI, 178p.

Poutouli W, Silvie P, Aberlenc HP. 2012. Hétéroptères Phytophages et Prédateurs d'Afrique de l'Ouest/ Phytophagous and predatory Heteroptera in West Africa. Ed. QUAE, CTA; 79.

Soyer D. 1942. Miride du cotonnier Creontiades pallidus Rambur Capsidae (Miridae). INEAC, Sér. Sci., 29: 15.

Stam PA. 1987. Creontiades pallidus (Rambur) (Miridae: Heteroptera), a pest on cotton along the Euphrates river and its effect on yield and control action threshold on Syrian Arab Republic. Tropical Pest Management, 33: 273-276.

Taylor THC. 1945. Lygus simonyi Reut., as a cotton capsid of Uganda. Bull. Entomol. Res., 36: 121-148.

Tingey WM, Pillemer E. Lygus bugs: Crop resistance and physiological nature of feeding injury. ESA Bulletin, 23: 177-287.

Tugwell P, Young SC, Dumas BA, Phillipis JR. 1976. Plant bugs in cotton: importance of infestation time, types of cotton injury and signification of wild host near cotton. Ark. Arg. Exp. Stat. Report, series, 227: 24. 